Zwei ungeklärte Todesfälle

\title{
Können wir die HPV-Impfung noch empfehlen?
}

Seit letztem Jahr empfiehlt die STIKO, Mädchen zwischen 12 und 17 Jahren Jahren gegen HPV zu impfen. Doch nun erlitten zwei gesunde Mädchen, eine 19-Jährige aus Österreich und eine 18-Jährige aus Deutschland, kurze Zeit nach einer HPV-Impfung einen plötzlichen Herzstillstand. Ist die Impfung schuld am Tod der Mädchen? MMW befragte dazu Prof. Dr. Johannes Löwer, den Präsidenten des Paul-Ehrlich-Instituts.

MMW: Ist ein Zusammenhang der beiden Todesfälle mit der HPV-Impfung nachweisbar bzw. auszuschließen?

Löwer: Die Untersuchungen der Staatsanwaltschaft und die Untersuchungen, die im Auftrag des Paul-Ehrlich-Instituts durchgeführt wurden, sind abgeschlossen. Für die beiden Todesfälle findet die medizinische Wissenschaft auf der Grundlage dessen, was sie heute weiß, keine Erklärung. Auch gibt es keinen Anlass zu glauben, dass die Impfung die Ursache für den Tod der beiden Mädchen ist.

MMW: Auch in den USA wurden einige Todesfälle in relativ kurzem zeitlichem Abstand nach einer HPV-Impfung bekannt. Beunruhigt Sie das?

Löwer: Wir haben uns die Unterlagen angesehen. Ein Großteil dieser Todesfälle ist nicht auswertbar. Bei einigen Todesfällen stellte man einen Myokardschaden fest, bei anderen deuten die Symptome darauf hin, dass es sich um Nebenwirkungen eines Kontrazeptivums handelt. Zumindest ein Fall könnte aber Ähnlichkeit mit den Fällen in Österreich und Deutschland haben. Ungeklärte Todesfälle kommen immer wieder vor, auch bei jungen Mädchen, und zwar ohne Zusammenhang zu einer Impfung. Inzwischen wurden weltweit etwa 20 Millionen Impfdosen verkauft. Da verwundert es nicht, dass sehr seltene Ereignisse wie diese Todesfälle hin und wieder in zeitlicher Nähe zur Impfung auftreten.

MMW: Wie sieht es mit anderen Nebenwirkungen aus?

Löwer: Das Paul-Ehrlich-Institut registrierte seit Einführung der HPV-Impfung 189
Verdachtsfälle von Nebenwirkungen. Kopfschmerzen sind dabei mit 13 Meldungen am häufigsten. Wie bei jeder Impfung wurden Erkrankungen gemeldet, bei denen theoretisch ein Zusammenhang zur Impfung denkbar wäre. Das ist der Fall, wenn ein immunologischer Mechanismus dahintersteckt. Bei der HPV-Impfung haben wir zwei Meldungen eines Guillain-Barré-Syndroms und drei Fälle einer idiopathischen thrombozytopenischen Purpura. Ob die Impfung tatsächlich dafür verantwortlich ist, ist schwer zu beurteilen. Alles andere sind Meldungen bekannter Impfnebenwirkungen, z. B. Reaktionen an der Einstichstelle.

MMW: Wurden mehr Nebenwirkungen gemeldet als bei anderen Impfstoffen? Löwer: Das ist schwer zu sagen, denn nach der Einführung eines Impfstoffs werden mögliche Nebenwirkungen meistens ge-

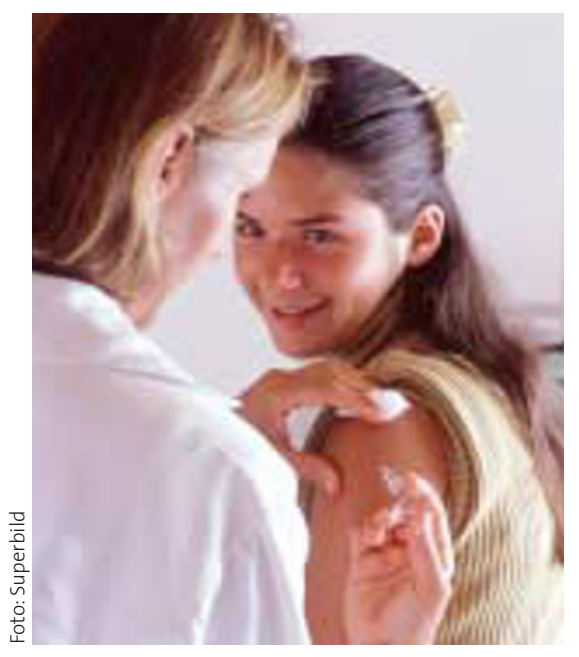

Impfung gegen HPV: Beim Paul-EhrlichInstitut wurden bisher 189 Verdachtsfälle von Impfnebenwirkungen registriert.

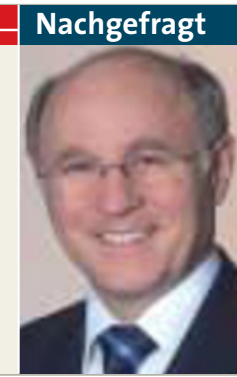

bei Prof. Dr. med. J. Löwer

„Bei 20 Mio. verkauften Impfdosen können ungeklärte Todesfälle auch in zeitlicher Nähe zur Impfung auftreten."

nauer beobachtet als bei älteren Impfstoffen. Aber ich habe nicht den Eindruck, dass bei der HPV-Impfung besonders viele oder ungewöhnliche Nebenwirkungen auftreten. Das sieht der Ausschuss für Humane Arzneimittel der EMEA genauso.

MMW: Als Ursache der Impfnebenwirkungen wurden Aluminiumverbindungen verdächtigt. Was halten Sie davon?

Löwer: Das ist völlig unverständlich. Aluminiumhydroxid wird seit Jahrzehnten als Adjuvans bei praktisch allen Totimpfstoffen eingesetzt. Warum das bei der HPVImpfung etwas Besonderes sein sollte, lässt sich nicht nachvollziehen.

MMW: Könnte es passieren, dass durch die Verdrängung der beiden Typen HPV 16 und 18 möglicherweise andere krebsauslösende HPV-Typen nachrücken?

Löwer: Ja, es gibt diese These. Entsprechende Studien sind auch bereits Teil des Risiko-Management-Plans. Vielleicht gibt es irgendwann auch einen Impfstoff gegen diese HPV-Typen. Es wird immer ein Kampf zwischen Mensch und Mikrobe bleiben.

MмW: Sollen Ärzte nun besser abwarten, bis weitere Daten vorliegen?

Löwer: Wir und auch die europäischen Behörden haben die Nutzen-Risiko-Beurteilung mit dem Wissen der beiden Todesfälle bereits neu durchdacht - mit dem Ergebnis, dass das Nebenwirkungsprofil dem aus den Zulassungsstudien bekannten entspricht. Da die Untersuchungen abgeschlossen sind, bringt es auch nichts, auf neue Erkenntnisse zu warten.

- Interview: Dr. Judith Neumaier

Haben Sie noch Fragen an Prof.Löwer? Sie erreichen inn über die MMW-Redaktion: Fax: 089-4372-1399

E-Mail: schumacher@urban-vogel.de 